\title{
EDITORIAL
}

\section{Gynecologic care after hematopoietic cell transplantation: a call to action to include gynecologists in the transplant team}

Bone Marrow Transplantation (2015) 50, 1-2; doi:10.1038/ bmt.2014.247; published online 3 November 2014

More than 30 different cancers, chronic diseases and hematologic conditions are currently treated by hematopoietic cell transplant resulting in about 50000 transplants a year. ${ }^{1,2}$ Cell-transplant physicians have successfully improved survivorship by decreasing the rate of relapse, improving engraftment, preventing or treating infections and screening for secondary cancers. ${ }^{3}$ A continuing challenge is chronic GVHD (cGVHD) and its long-term sequelae. ${ }^{4}$ The pathophysiology of CGVHD remains one of the most poorly understood complications of hematopoietic cell transplantation $(\mathrm{HCT})$. Thus, for now, the focus remains on early recognition of cGVHD and then initiating treatment in hopes of minimizing its long-term effects. In addition, the transplant process results in many physiological changes including gonadal failure that alter the quality of life. These changes differ depending on the gender and age of the patient.

In this month's journal, Dr Frey Tirri et al. ${ }^{5}$ have accomplished something unusual in the care of women after cell transplant. Their regional cell transplant organization in Switzerland, Germany and Austria has developed a consensus statement for the gynecologic care of women. Their paper is a comprehensive review of the current knowledge regarding gynecologic health and provides recommendations for post-transplant gynecologic care with the goal to diagnose, to prevent and then treat female genital cGVHD. They set forth the importance of fertility preservation counseling, options for hormonal treatment for premature ovarian failure and for treatment of diminished libido. They also address the assessment of pediatric patients not only for signs of CGVHD but also for pubertal status. If these young girls do not produce estrogen after transplant, this lack of estrogen can prevent the development of secondary sex characteristics.

Why is this important? Within post-transplant care, genital cGVHD is likely common but underreported. In part, this underreporting arises because women rarely report genital symptoms to their health providers and their health providers rarely ask. Genital cGVHD significantly affects intimacy and, in turn, reduced intimacy affects the quality of life. In fact, sexual quality of life is reduced for women soon after transplant, which is not a surprise given that the first year after transplant is often marked by substantial health challenges. This journey to health takes a profound toll on patients and their significant others. What is surprising is that male patients recover their sexual health, but female patients often do not. ${ }^{6}$ In part, this difference may be related to the late onset of female genital CGVHD, which occurs as early as 7-10 months but can often develop years later. ${ }^{7}$

Dr Frey Tirri et $a l^{5}$ importantly emphasize that the diagnosis of genital cGVHD cannot be based on symptoms alone. In fact, women with symptoms like dyspareunia or vulvovaginal pain and discomfort on pelvic exam may or may not have genital cGVHD. At physical examination, the differential diagnosis of clinical signs warrants investigation to exclude infections, drug or allergic reactions or genital dysplasia. At times, histology is helpful in excluding Human papillomavirus (HPV)-related neoplasia, but is not required for the diagnosis of genital cGVHD. When lichen planus-like features or vaginal scarring, diagnostic signs of genital CGVHD, are observed on gynecologic examination, specific treatment can be initiated. Photo documentation is helpful in assessing response to treatment and is more helpful than performing repeated biopsies.

The authors present several key principles for treatment of genital cGVHD. ${ }^{5}$ These principles include: (1) reducing irritation of genital skin; (2) using topical estrogen treatment in addition to systemic hormone replacement; (3) achieving control of GVHDrelated inflammation; (4) once the genital cGVHD is treated, substituting topical calcineurin inhibitors rather than using topical corticosteroids for the long term; (5) assessing response to treatment by photo documentation; (6) using mechanical measures to improve vaginal narrowing.

Dr Frey Tirri et $a l^{5}$ also address other aspects of comprehensive health care for women. Not only is genital cGVHD a significant problem, but gonadal failure is also more common in women than men and often is not addressed as a health issue. Importantly, genital atrophy due to estrogen deficiency may be a confounding factor for female genital CGVHD. They recommend that women with premature ovarian failure arising from transplant would likely benefit from hormone therapy till the median age of menopause unless there are contraindications like thrombophilias or hormone-sensitive cancers. They also recommend that the gynecological examination includes cervical cytology screening once a year with follow-up colposcopy and biopsy for either abnormal cytology or suspicious genital lesions. They also consider offering HPV vaccination to women after transplantation. ${ }^{8}$

The authors highlight that by consistently diagnosing, documenting and treating genital CGVHD, we will not only determine its prevalence, but also can develop consistent strategies to prevent or treat its long-term effects. That approach will perhaps lead to insights into its pathophysiology. Ultimately successfully preventing, diagnosing and treating genital cGVHD in women after transplant will improve their quality of life. Women benefit from an assessment by a gynecologist with knowledge in transplant care at least yearly. To facilitate early diagnosis of genital cGVHD, at a minimum, women presenting with other signs of cGVHD should be asked about genital symptoms like pain or burning. Those women with genital symptoms or worsening systemic CGVHD may also have genital CGVHD and warrant examination by a gynecologist with expertize in diagnosis and treatment of cGVHD. Once genital cGVHD is diagnosed, patients benefit from close follow-up until the signs and symptoms are treated.

Dr Frey Tirri et $a .^{5}$ underscore the necessity for regular gynecologic care and screening programs for women after HCT. In fact, gynecologists have a role in addressing reproductive health concerns along the entire spectrum of care for women undergoing HCT and are critical members of the transplant team. Before transplant, gynecologists serve as valuable consultants in addressing the appropriate timing of initiating menses suppression agents like gonadotropin-releasing hormone agonists for maximal benefit, future fertility, contraception, therapeutic amenorrhea and peri-transplant reproductive health concerns. After transplant through long-term survivorship care, 
gynecologists can continue to address issues such as revaccination for HPV, screening for genital cancer and cGVHD, hormone replacement therapy, fertility and sexuality. In short, their paper is a call to action for the transplant community and gynecologists alike.

\section{CONFLICT OF INTEREST}

The author declares no conflict of interest.

\section{ACKNOWLEDGEMENTS}

This paper was supported in part by the Intramural Research Program of the National Institutes of Health and the Program in Reproductive and Adult Endocrinology, Eunice Kennedy Shriver NICHD, Clinical Center, National Institutes of Health.

P Stratton

Gynecology Consult Service, Program in Reproductive and Adult Endocrinology, Eunice Kennedy Shriver National Institute of Child Health and Human Development (NICHD), Bethesda, MD, USA

E-mail: ps79c@nih.gov

\section{REFERENCES}

1 Milroy CL, Jones KP. Gynecologic care in hematopoietic stem cell transplant patients: a review. Obstet Gynecol Surv 2010; 65: 668-679.

2 Copelan EA. Hematopoietic stem-cell transplantation. N Engl J Med 2006; 354: 1813-1826.

3 Porta F, Locatelli F, Burgio GR. Hematopoietic stem cell transplantation: 40 years of continuous progress and evolution. Haematologica 2008; 93 1607-1610.

4 Garnett C, Apperley JF, Pavlu J. Treatment and management of graft-versus-hos disease: improving response and survival. Ther Adv Hematol 2013; 4: 366-378.

5 Frey Tirri B, Häusermann P, Bertz H, Greinix H, Lawitschka A, Schwarze C-P et al. Clinical guidelines for gynecologic care after hematopoietic SCT. Report from the international consensus project on clinical practice in chronic GVHD. Bone Marrow Transplant 2015; 50: 3-9.

6 Shanis D, Merideth M, Pulanic TK, Savani BN, Battiwalla M, Stratton P. Female longterm survivors after allogeneic hematopoietic stem cell transplantation: evaluation and management. Semin Hematol 2012; 49: 83-93.

7 Stratton P, Turner ML, Childs R, Barrett J, Bishop M, Wayne AS et al. Vulvovaginal chronic graft-versus-host disease with allogeneic hematopoietic stem cell transplantation. Obstet Gynecol 2007; 110: 1041-1049.

8 Tedeschi SK, Savani BN, Jagasia M, Engelhardt B, Anasetti C, Barrett AJ et al. Time to consider HPV vaccination after allogeneic stem cell transplantation. Biol Blood Marro w Transplant 2010; 16: 1033-1036. 